\title{
Study of Nonlinear Mode Coupling During Neoclassical Tearing Modes Using Bispectrum Analysis
}

\author{
Raju $\mathrm{D}^{1}$, Lister J B and Sauter O \\ Centre de Recherches en Physique des Plasmas, CRPP - EPFL, Association \\ EURATOM-Confédération Suisse, PPB-Ecublens, 1015 Lausanne, Switzerland
}

\begin{abstract}
It is sometimes observed that several Neoclassical Tearing Modes (NTMs) are present at the same time. This can happen both at the mode onset of a new NTM or during the nonlinear saturated phase of a given mode. In order to analyse whether nonlinear mode coupling plays any role before or after the onset of NTMs, we use a data analysis tool known as the bispectral technique. This technique has been used for identifying the nonlinear three wave interactions in turbulent plasmas. We have used this technique for the study of NTMs in the Tokamak à Configuration Variable (TCV) and in the Joint European Torus (JET). The analysis during the saturated phase of a $3 / 2$ NTM in a JET discharge suggests a strong coupling between the $3 / 2,4 / 3$ and $7 / 5$ modes. This coupling could be responsible for the observed stabilisation effect of the $4 / 3$ mode on the $3 / 2$ mode [Sauter $\mathrm{O}$ et al, Plasma Physics Contr. Fus. 44 (2002) 1999]. It also demonstrates that this analysis tool can be used to identify high $\mathrm{m} / \mathrm{n}$ modes, beyond the capabilities of the standard method using poloidal or toroidal probe arrays. As this technique allows a more accurate evaluation of the existence of nonlinear mode coupling, it has been used to study and confirm that no significant nonlinear coupling occured to trigger two specific NTM onset cases in TCV and JET, where a classical tearing mode and a crash after a long sawtooth period are responsible for the triggering of the necessary seed islands, respectively.
\end{abstract}

Submitted to: Plasma Phys. Control. Fusion

E-mail: raju@ipr.res.in

1 Present adress: Institute for Plasma Research, Bhat, Gandhinagar, Gujarat-382 428, INDIA 


\section{Introduction}

Neoclassical tearing modes (NTMs) are the main limitation to achieve high normalised plasma pressure in the standard high confinement mode in tokamaks. In several experiments $[1,2,3]$, it was observed that the performance in terms of total plasma stored energy and neutron yield deteriorate with the onset of NTMs. These modes have low poloidal $(m)$ and toroidal $(n)$ mode numbers. Typically $m / n=3 / 2,4 / 3$ or $2 / 1$. They are driven by the flattening of the pressure profile across the existing island [4]. At small island sizes other effects tend to oppose this drive, leading to the requirement of a minimum size of seed islands. Such seed islands are usually provided by the instabilities and perturbations associated with sawteeth, fishbones or ELMs [4].

The $3 / 2$ NTM is often observed at intermediate $\beta_{N}$ and degrades the thermal energy confinement time by typically $10-20 \%$, where $\beta_{N}$ is the ratio of the plasma pressure to the confining magnetic pressure normalised to $I / a B$ ( $I[\mathrm{MA}]$ the plasma current, $a[\mathrm{~m}]$ the minor radius and $B[\mathrm{~T}]$ the magnetic field). Usually at higher $\beta_{N}$, the more dangerous 2/1 NTM is observed, which can significantly deteriorate the plasma performance and sometimes lead to a disruption. The scaling of $\beta_{N}$ at the NTM onset with the normalised ion Larmor radius, $\rho *=\rho / a$, gives rise to particular concern for next generation tokamaks such as ITER-FEAT [5] which will have a high magnetic field and a low $\rho *$. Several studies show an approximate linear scaling in this parameter [3] and this scaling points to a very low $\beta_{N}$ threshold for large tokamaks like ITER-FEAT.

The trigger mechanism of the seed island is not always clear at present. It has been shown in the Tokamak à Configuration Variable (TCV) that classical tearing modes can generate the seed islands required for NTMs to be destabilised further by the perturbed bootstrap current [6]. On the other hand it has been observed recently in the Joint European Torus (JET) that by inducing long sawtooth periods, NTMs can be destabilised at very low plasma beta values [7]. These latter results suggest that seed islands might be relatively easily triggered by the long sawtooth period expected in burning plasmas like ITER-FEAT due to alpha-particle stabilisation [8]. It has also been observed during a long phase with stationary saturated 3/2 NTMs that 4/3 modes can stabilise the $3 / 2$ mode, demonstrating strong mode coupling [9, 10]. In JET discharges there are cases with matching of the $3 / 2,4 / 3$ and $1 / 1$ frequencies, or between the $3 / 2$ and two other modes, and nonlinear mode coupling can be responsible for driving the necessary seed island. It has been shown recently that such a coupling is sufficient in JET conditions to explain the $3 / 2$ mode onset as a result of a three wave coupling with $4 / 3$ and $1 / 1$ modes [11]. A study of a range of JET discharges shows that in some cases the expected frequency match to allow nonlinear mode coupling does not occur (or the $4 / 3$ mode is absent altogether) and therefore in these cases neither the crash itself, as with long sawtooth period, nor nonlinear mode coupling can explain the NTM trigger $[12]$.

In order to be able to quantify when nonlinear mode coupling is at play, we have used a data analysis tool known as the bispectral technique. This technique has previously 
been used for identifyng the nonlinear three wave interactions in turbulent plasmas [13]. The method allows an accurate check on the three wave frequency criteria and phase coherence. We first use this tool to analyse onset cases where no nonlinear mode coupling is expected $[6,7]$ in order to verify this assumption and to demonstrate the use of this diagnostic tool in simple cases. We then study a case during the saturated phase of a $3 / 2$ NTM, where a $4 / 3$ mode has been observed to stabilise the $3 / 2$ mode while many other modes are present [10]. The bispectrum shows in this case that there is actually a continuous nonlinear coupling between the modes $4 / 3,3 / 2$ and $7 / 5$.

\section{Bispectral analysis}

For nonlinear three wave interactions, the wave frequencies $\left(f_{1}, f_{2}, f_{3}\right)$ and wave numbers $\left(\mathbf{k}_{1}, \mathbf{k}_{2}, \mathbf{k}_{3}\right)$ must satisfy the selection rules of resonance conditions: $f_{1}+f_{2}=f_{3}$ and $\mathbf{k}_{1}+\mathbf{k}_{2}=\mathbf{k}_{3}$. In fluctuating data, such selection rules are often satisfied. Note also that the Fourier transform involved in this analysis cannot determine the sign of the frequency when applied to magnetic data measurements as in this paper. Therefore the "resulting" mode could also be $f_{2}$, with $f_{2}=\left(-f_{1}\right)+f_{3}$. If $f_{3}$ (or $f_{2}$ ) is an independent mode, the question arises how to discriminate with nonlinear mode coupling. Bispectrum, a higher order spectrum, permits the distinction of the spontaneously excited normal modes from the coupled modes by measuring the degree of phase coherence between the modes. The bispectrum is defined as:

$$
B\left(f_{1}, f_{2}\right)=E\left[X\left(f_{1}\right) X\left(f_{2}\right) X^{\star}\left(f_{1}+f_{2}\right)\right],
$$

where E denotes the Expected Value, $\mathrm{X}(f)$ is the Fourier transform of $\mathrm{x}(\mathrm{t})$ and * represents the complex conjugate. The above equation clearly indicates that the bispectrum will be zero unless three waves at frequencies $f_{1}, f_{2}$ and $f_{1}+f_{2}$ are present in the fluctuating data with phase coherency between the waves. For spontaneously excited non-interacting modes, the phases would be random and therefore the statistical averaging will take a zero value. A quantitative measure of the phase coherence is made by computing the bicoherence, defined in terms of the bispectrum:

$$
b^{2}\left(f_{1}, f_{2}\right)=\frac{\left|B\left(f_{1}, f_{2}\right)\right|^{2}}{P\left(f_{1}\right) P\left(f_{2}\right) P\left(f_{1}+f_{2}\right)},
$$

where $P(f)$ is the auto-power spectrum. In principle, when a nonlinear interaction is taking place then $b\left(f_{1}, f_{2}\right)$ will take a value close to unity, indicating a high degree of phase coherence. For spontaneously generated modes, this value will be close to zero. In analysing real data one expects values greater than 0.5 to suggest significant coupling. Note also that this method is valid only if the mode frequencies do not vary too much in the time interval analysed. The approximate variance of the bicoherence estimator is defined as [13]:

$$
\operatorname{var}\left(b^{2}\right) \approx(M / 2 N)\left[1-b^{2}\left(f_{1}, f_{2}\right)\right]
$$

where $N$ is the number of total data points and $M$ is the number of data records into which the data time series is divided (of say, 256 data points each). 
The sampling rates used for the Mirnov coil data in TCV and JET are $125 \mathrm{kHz}$ and $250 \mathrm{kHz}$ respectively. We use at least 65 records each consisting of 128 data points in the case of TCV and about a similar number of records each consisting of 256 data points in the case of JET. The fast Fourier transform algorithm is used to generate Fourier components for each record. Bicoherence is then computed as described above. In this way the variance in this analysis is about 0.015 giving an accuracy of about $1 \mathrm{kHz}$ in the frequency resolution. It should be noted that in the case of MHD modes in tokamaks, the frequency measured in the laboratory frame are Doppler shifted with respect to the mode frequency in the plasma frame. The Doppler shift is essentially proportional to the toroidal mode number and it obeys by definition the same selection rule, namely $n_{1}+n_{2}=n_{3}$. Therefore if the Doppler shift is much larger than the mode frequencies and if the modes are at nearby radii or the rotation profile is nearly constant, it is difficult to extract the relevant data accurately and one could easily find coherent modes with matching frequencies. This means that one needs an accuracy of at least the diamagnetic frequencies, which is expected to be of the order of $1 \mathrm{kHz}$ in both JET and TCV. This might limit the application of such studies when there is a too small time window where the modes are present or with constant frequencies. Another limit is of course the minimum island size detectable by the external magnetic coils, for example of the order of $1 \mathrm{~cm}$ in JET. However this method can detect the level of bicoherence even if the signal is relatively small, as will be seen below.

Since the bispectrum has certain symmetry properties $[14,15]$ and the technique is limited by the Nyquist frequency, $f_{N y q}$, we compute the bicoherence in two triangular regions of the $f_{1}, f_{2}$ plane:

(a) the sum interaction region:

$$
0 \leq f_{1} \leq f_{N y q} \quad ; \quad f_{2} \leq f_{1} \quad ; \quad f_{1}+f_{2} \leq f_{N y q}
$$

(b) the difference interaction region:

$$
0 \leq f_{1} \leq f_{N y q} \quad ; \quad-f_{N y q} \geq f_{2} \geq 0 \quad ; \quad f_{1}+f_{2} \leq f_{N y q}
$$

\section{Island width time evolution}

We first discuss a TCV discharge, \#15963, where electron cyclotron heating and current drive are applied, between $0.6 \mathrm{~s}$ and $2.2 \mathrm{~s}$, and trigger $3 / 2$ and $2 / 1$ NTMs. The magnetic data and corresponding spectrogram are shown in Fig. 1. It has been shown in Ref. [6] that the 2/1 NTM is first destabilised by the classical $\Delta^{\prime}>0$ and then the bootstrap drive dominates once the island has grown to a sufficient size. However a small $3 / 2$ mode is also destabilised before the $2 / 1$ mode and we have used the bispectrum analysis to check if it plays a role in the $2 / 1$ evolution, coupled to a $1 / 1$ mode for example. The bicoherence results obtained at different time intervals are shown in Fig. 2, where we have also marked a few extra indications to show the information one can obtain from these figures. The corresponding intervals are marked on Fig. 1. The left triangular region (the difference interaction region, Eq. (5)), $f_{1}$ versus $f_{2}<0$, gives the coupling 
for $f_{3}=f_{1}-\left|f_{2}\right|$, while the right triangular part (the sum interaction region, Eq. (4)), $f_{1}$ versus $f_{2}>0$, gives the $f_{3}=f_{1}+\left|f_{2}\right|$ coupling. Coupled modes should exhibit peaks of bicoherence value greater than 0.5 in such a contour plot as mentioned in Sec. 2. No significant peaks are observed in Figs. 2(a and b) and therefore no evidence of nonlinear mode coupling is found before the mode onsets. Just after the 2/1 mode $(f \approx 4.5 \mathrm{kHz})$ onsets and in the saturated phase, a strong coupling with the $4 / 2$ mode harmonic $(f \approx 9 k H z)$ is observed as expected. The scale shows that these modes have a bicoherence value of $0.7-0.8$.

Harmonic coupling is observed when a peak lies near the $f_{1}=f_{2}$ dashed diagonal line marked in Fig. 2(c) in the sum interaction region, as it means that nonlinear coupling occurs with $f_{3}=f_{1}+f_{2}=2 f_{1}$. Similarly, coupling between the $4 / 2$ mode at $f_{1} \approx 9 k H z$ and the $2 / 1$ mode at $f_{2} \approx-4.5 k H z$ is shown by the peak marked in the difference interaction region in Fig. 2(c). Therefore harmonic coupling will show up in the difference interaction region as peaks along the dashed line marked in Fig. 2(d): $f_{1}=-2 f_{2}$. Therefore only modes which do not lie on these two dashed lines are of interest as they show non-harmonic nonlinear mode coupling. Since no such coupling is found in this case, as expected from direct Fourier analysis, nonlinear mode coupling does not play a role in destabilising the $3 / 2$ NTM.

In the case of long sawtooth periods induced by fast particle stabilisation with ICRF heating, NTMs are triggered at the sawtooth crash which can trigger several modes at once [7]. The raw data and the spectrogram are shown in Fig. 3 near the sawtooth crash which happens at 18.75s. No significant interaction is observed before the crash and one expects no mode coupling. This is confirmed by the bispectrum analysis which shows no significant peaks. Immediately after the crash several modes exist, as shown in Fig. 3, and, apart from harmonic coupling, a nonlinear three wave interaction with modes at frequencies 12,15 and $27 \mathrm{kHz}$ is observed with a bicoherence value of about 0.7 (Fig. $4(\mathrm{a}))$. Note that the harmonic coupling at $12 \mathrm{kHz}$ is slightly stronger, at about 0.8 . The mode at $12 \mathrm{kHz}$ is identified as a $3 / 2 \mathrm{NTM}$ and at $15 \mathrm{kHz}$ as a $4 / 3$ mode. Therefore the mode at $27 \mathrm{kHz}$ is found to be a $7 / 5$ mode to satisfy $\mathbf{k}_{3}=\mathbf{k}_{1}+\mathbf{k}_{2}$. However both higher modes are stable and disappear rapidly, leaving "only" the $3 / 2$ mode and its harmonics. Note that very strong harmonic coupling is observed as shown in Fig. 4(b), as the 3/2 couples with the second, $6 / 4$ at $20 \mathrm{kHz}$, and the third harmonics, $9 / 6$ at $30 \mathrm{kHz}$, and so on. These couplings are observed as peaks along the vertical line $f_{2}=+10 \mathrm{kHz}$, that is at $\left(f_{2}=10 \mathrm{kHz}, f_{1}=p * 10 \mathrm{kHz}\right)$ in the sum region, where $p$ is the harmonic number. In the difference interaction region, this leads to peaks at $\left(f_{2}=-10 k H z, f_{1}=p * 10 k H z\right)$, and on the lines $f_{1}=-p /(p-1) f_{2}$. Note that the $6 / 4$ mode itself is strong enough to exhibit harmonic coupling as shown by the peaks at $\left(f_{2}=20 \mathrm{kHz}, f_{1}=20 \mathrm{kHz}\right)$ in the sum region and at $\left(f_{2}=-20 k H z\left(-\frac{6}{4}\right), f_{1}=40 k H z\left(\frac{12}{8}\right)\right)$ in the difference region. Finally there is even a small coupling between the $9 / 6$ and the $6 / 4$, bicoherence $\approx 0.4$, contributing to the $15 / 10$ mode at $50 \mathrm{kHz}$. As each frequency corresponds to a specific mode, the different couplings are readily obtained from Fig. 4(b) using the mode labels instead of the frequency values for the $y$ - and x-axis. For example the four peaks at 
$\mathrm{y}=\frac{9}{6}$ denote the $\frac{9}{6}-\frac{6}{4}, \frac{9}{6}-\frac{3}{2}, \frac{9}{6}+\frac{3}{2}$ and $\frac{9}{6}+\frac{6}{4}$ couplings (using the labels on $\mathrm{x}$-axis for the second mode). In this way we can explain all the peaks in Fig. 4. It also shows that the bispectrum analysis is a very useful tool to determine the $m$ and $n$ values of high frequency modes, which high $m, n$ values are otherwise difficult to diagnose with great confidence. In JET, fast magnetic data are acquired in short intervals of typically 100$200 \mathrm{~ms}$. In these intervals one can obtain $m, n$ values up to $m \sim 4, n \sim 5$. One interval is around $19 \mathrm{~s}$ and confirms the $n=2$ and $n=4$, but only $m=3$ for the $3 / 2$ mode can be extracted..

Finally we have chosen another JET discharge, \#53290, with a saturated 3/2 NTM and where many modes are at play. In this discharge, while the input power is slowly decreasing to analyse the marginal beta limit, a stationary $3 / 2$ island width is observed to strongly decrease in size at the onset of a $4 / 3$ mode [10]. This $4 / 3$ mode is triggered by a sawtooth crash and is stabilised by the next sawtooth crash. Consequently the $3 / 2$ mode increases again once the $4 / 3$ mode has disappeared. This effect has been successfully modelled using an ad-hoc direct coupling included in the modified Rutherford equation for the $3 / 2$ mode as an additional constant stabilising term, $\Delta_{4 / 3}^{\prime}<0$ [10]. The bispectrum analysis is used to analyse the different mode interactions more accurately. The spectrogram around the two sawtooth crashes at $21.78 \mathrm{~s}$ and $22.45 \mathrm{~s}$ is shown in Fig. 5 and the corresponding bicoherence data are presented in Fig. 6. Just before the first sawtooth crash, we observe mainly the harmonics $3 / 2$ and $6 / 4$ coupling, with a bicoherence value of 0.8 , and a smaller $1 / 1$ and $2 / 2$ coupling, even though the $1 / 1$ mode is strong. Just after the sawtooth crash, more mode interactions are observed in addition to the strong harmonic coupling of the $3 / 2$ at about $8 \mathrm{kHz}$. In particular, nonlinear coupling between the $3 / 2,4 / 3(12 \mathrm{kHz})$ and the $7 / 5(20 \mathrm{kHz})$ is observed. The possibility of such mode coupling between saturated modes is consistent with simulations presented in Ref. [16]. A small interaction between the $4 / 3,3 / 2$ and $1 / 1$ modes is also observed at $(-8 \mathrm{kHz}, 12 \mathrm{kHz})$, and $(-4 \mathrm{kHz}, 12 \mathrm{kHz})$ in the difference region and at $(4 \mathrm{kHz}, 8 \mathrm{kHz})$ in the sum region. However this coupling is intermittent as seen in the following plots. Note nevertheless that coupling with the $1 / 1$ mode is observed in Fig. 6(b and d) even though the mode amplitude is small as seen in Fig. 5. On the other hand the 3/2,4/3 and 7/5 interaction persists and increases as the bicoherence value goes from about 0.4 (Fig. 6(b)) to about 0.8 (Figs. 6(d) and (e)). We have labeled the different interactions, for example $7 / 5-4 / 3$ or $3 / 2+3 / 2$, where the first mode correspond to the $\mathrm{y}$-axis, $f_{1}$, and the second to the x-axis, $f_{2}$. Thanks to the strict rule on $m, n$ for nonlinear mode coupling, one can identify all the peaks observed in these contour plots of the bicoherence. Note that there is no significant differences with and without the strong $1 / 1$ mode which exists before each sawtooth crash and in particular during the time interval considered in Fig. 6(e). This confirms that the $4 / 3$ and $3 / 2$ interact dominantly with the $7 / 5$ and not the $1 / 1$. The increase in the bicoherence of the mode coupling suggest that the $7 / 5$ mode is driven by the $4 / 3$ and $3 / 2$ modes and could explain partly why the $3 / 2$ mode decreases in amplitude.

We show in Fig. 7 the time evolution of the square root of the magnetic perturbation 
measurement at the edge for these three modes, as well as their respective frequencies. Note that $f_{3 / 2}+f_{4 / 3}$ (dashed red line in Fig. $(7 \mathrm{~b})$ ) does coincide exactly with the full time evolution of $f_{7 / 5}$ whenever $\delta B_{7 / 5}$ can be measured. As mentioned before, the $3 / 2$ island width steadily decreases as soon as the $4 / 3$ and $7 / 5$ modes exist (Fig. (7a)). The fact that the $7 / 5$ has a constant amplitude up to the next sawtooth crash could explain the constant stabilisation of the $3 / 2$ mode. After the second crash, $22.45 \mathrm{~s}$, both $4 / 3$ and $7 / 5$ modes decay rapidly and the $3 / 2$ mode recovers a positive growth. This is confirmed by the bispectrum analysis, Fig. (6f), which exhibits only $3 / 2$ and $6 / 4$ harmonic coupling after the second sawtooth crash at $22.45 \mathrm{~s}$.

\section{Discussion}

The bispectral technique can be a very useful tool to identify nonlinear three wave mode coupling and to determine whether they can be responsible for triggering NTMs. It has been confirmed that the $3 / 2$ mode is not involved in the 2/1 NTM destabilisation in ECCD experiments in TCV. Similarly no significant mode coupling has been observed prior to a long sawtooth period in JET after which multiple modes are triggered by the crash. The systematic study of discharges where long sawtooth periods are not present and therefore do not dominate the seed island mechanisms is left for future studies.

During a saturated phase of a $3 / 2$ NTM, strong nonlinear mode coupling, with a bicoherence up to 0.6-0.8, has been observed between the $3 / 2,4 / 3$ and $7 / 5$ modes in JET discharges where strong $3 / 2$ stabilisation due to the presence of the $4 / 3$ mode has been observed, similarly to the situation simulated in Ref. [16]. The time evolution of the bicoherence suggests that the $7 / 5$ mode is driven by the $3 / 2$ and the $4 / 3$ modes, and the constant size of the magnetic perturbation measured at the edge could explain the constant "damping" observed on the $3 / 2$ mode [10].

It has also been shown that this technique is very useful to determine high $m, n$ values of specific modes where standard techniques cannot be applied. It is therefore an additional MHD spectroscopy tool and should prove valuable in future studies.

\section{Acknowledgments}

One of the author (OS) would like to thank Prof. A. Fasoli for useful discussions. This work was partly supported by the Fonds National Suisse de la Recherche Scientifique and conducted in part under the European Fusion Development Agreement.

\section{References}

[1] Chang Z et al, Phys. Rev. Lett., 74 (1995) 4663.

[2] Gude A et al, Nucl. Fusion, 39 (1999) 127.

[3] Buttery R J et al, Plasma Phys. Contr. Fusion 42, B61 (2000).

[4] Sauter O et al, Phys. Plasmas, 4 (1997) 1654.

[5] Aymar R. et al, Nucl. Fusion 41, 1301 (2001). 
[6] Reimerdes H et al, Phys. Rev. Lett. 88 (2002) 105004; PhD Thesis, Nr. 2399, Lausanne, EPFL; Lausanne report, CRPP, LRP 700/01.

[7] Sauter O et al, Phys. Rev. Lett. 88, 105001 (2002).

[8] Porcelli F et al, Plasma Phys. Contr. Fusion 38, 2163 (1996)

[9] Gunter S et al, Phys. Rev. Lett. 87, 275001 (2001).

[10] Sauter O et al, Plasma Phys. Contr. Fus. 44, 1999 (2002).

[11] Nave M F F et al, in Proc. 27th Eur. Phys. Soc. Conf., Budapest (2000), Europhysics Abstracts Vol. 24B 1100 (2000); submitted to Nuclear Fus.

[12] Buttery R J et al, accepted for publication in Nucl. Fus.

[13] Hinich M J and Clay C S, Rev. Geophys., 6 (1968) 347.

[14] Lii K S et al, J. Fluid Mech., 77 (1976) 45.

[15] Hasselmann K, Munk W H, and MacDonald G J F, in Time series analysis, edited by M. Rosenblatt (Willey, New York, 1963), p. 125.

[16] Coelho R et al, Phys. Plasmas 6, 1194 (1999). 

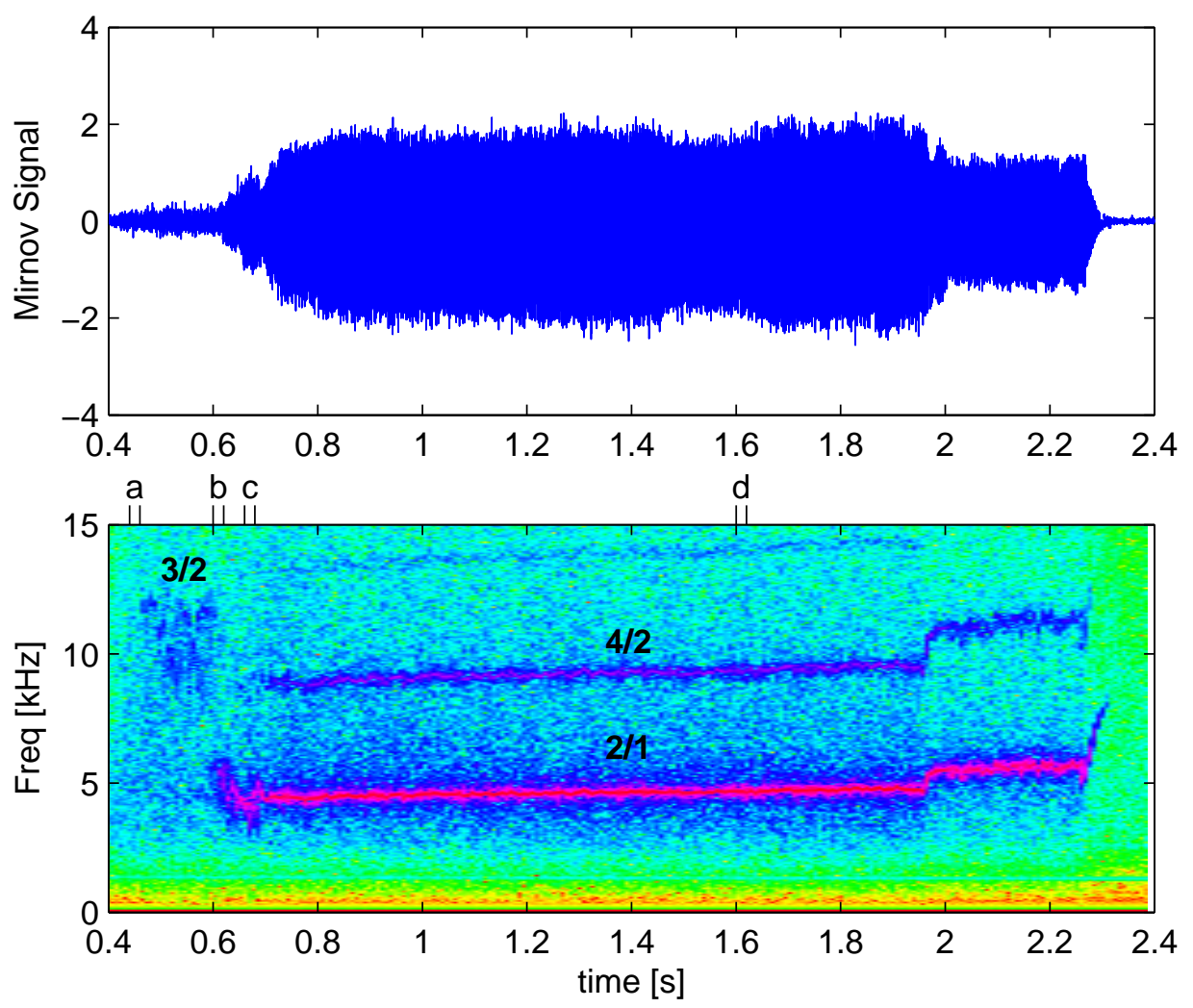

Figure 1. Mirnov coil signal (top curve) and the corresponding spectrogram between $0.4 \mathrm{~s}$ and $2.0 \mathrm{~s}$ of a TCV discharge discussed in Ref. [6]. The time intervals a, b, c and d correspond to those used for the bicoherence analyses in Fig. 2.
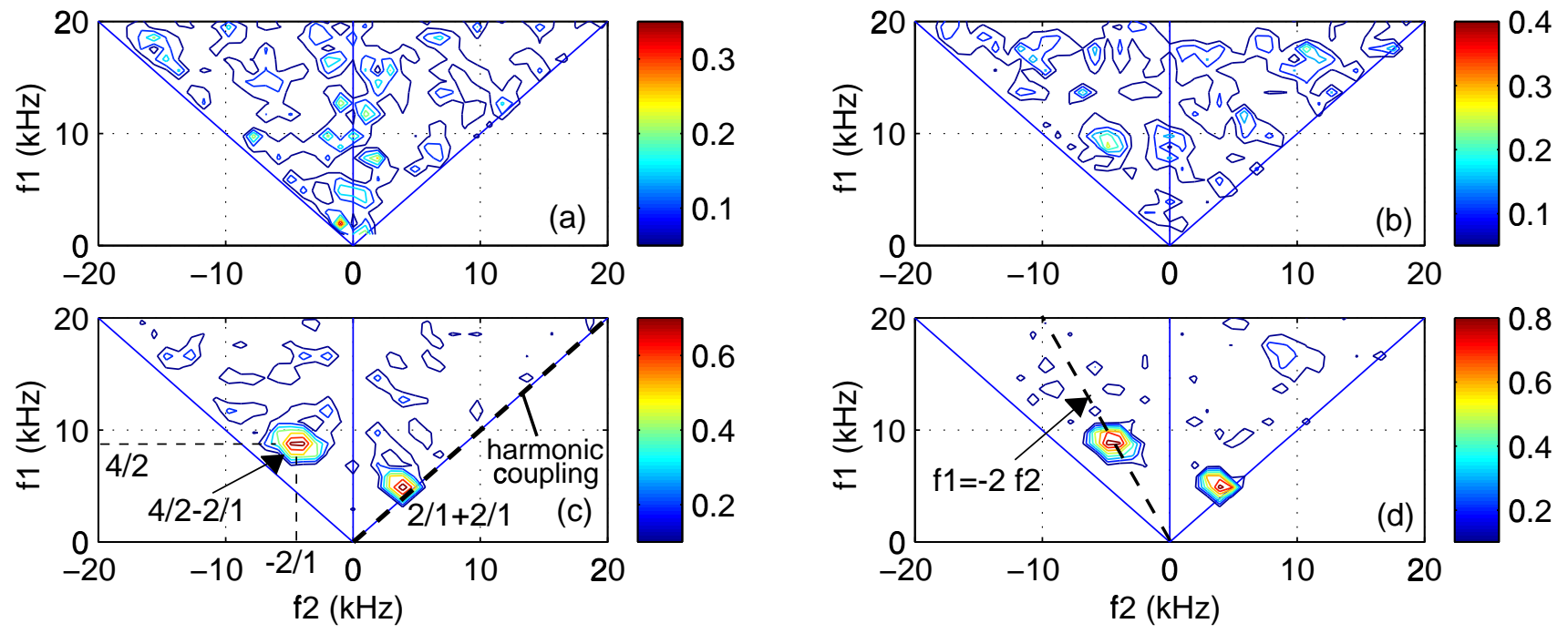

Figure 2. Bispectral analysis of the discharge shown in Fig. 1. The scale shows the bicoherence value as described by Eq. (2) (similarly in Fig. 4 and 6). Time slice between (a) $0.44 \mathrm{~s}$ and $0.46 \mathrm{~s}$, (b) $0.6 \mathrm{~s}$ and $0.62 \mathrm{~s}$, (c) $0.66 \mathrm{~s}$ and $0.68 \mathrm{~s}$ and (d) $1.6 \mathrm{~s}$ and $1.62 \mathrm{~s}$. These time intervals are marked in Fig. 1. 


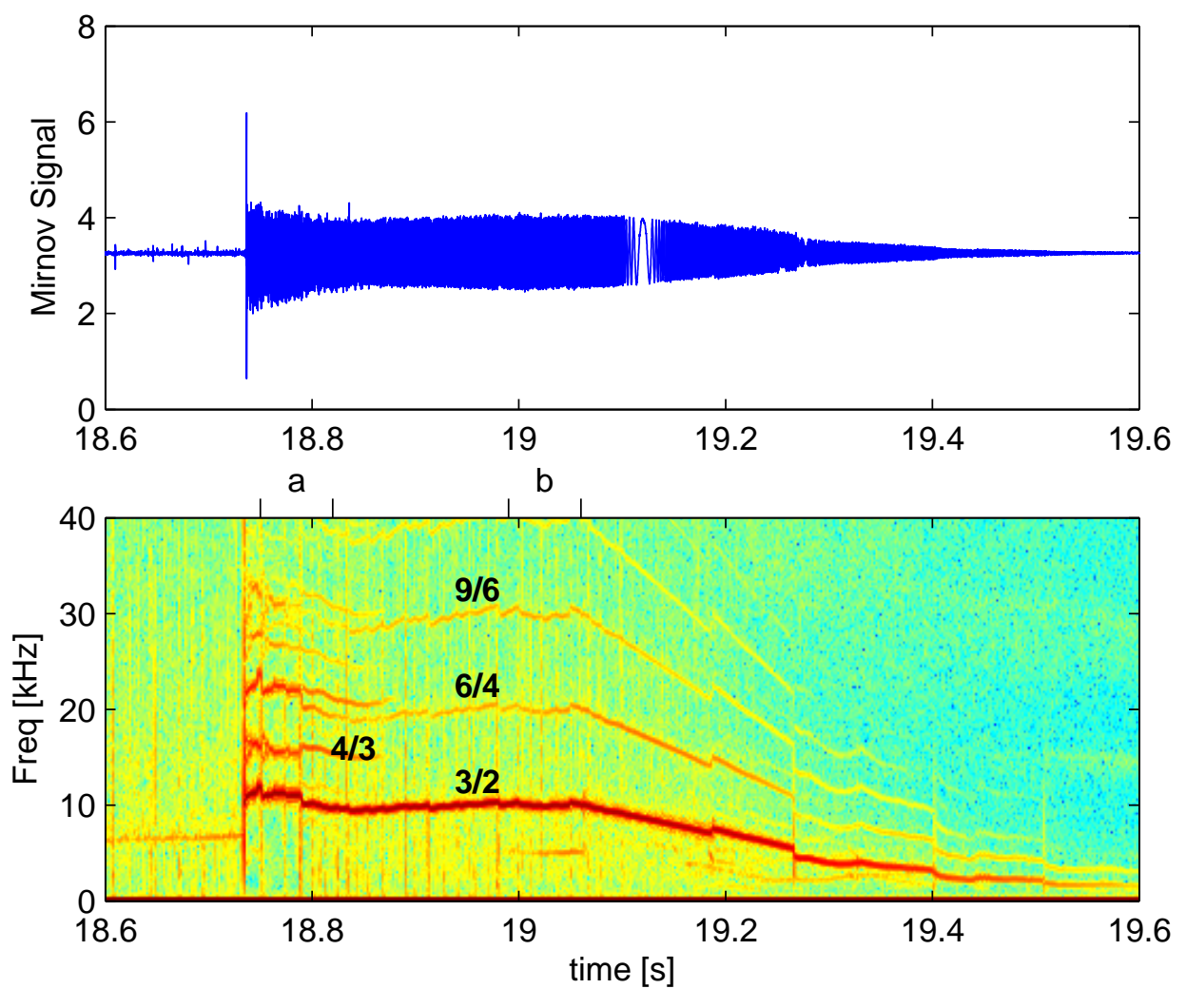

Figure 3. Mirnov coil signal (top curve) and the corresponding spectrogram between 18.0s and 20.0s for a JET discharge, \#53285, at 3.3T/3.3MA discussed in Ref. [7]. Bicoherence shown in Fig. 4 are calculated for the marked intervals $\mathrm{a}$ and $\mathrm{b}$. 

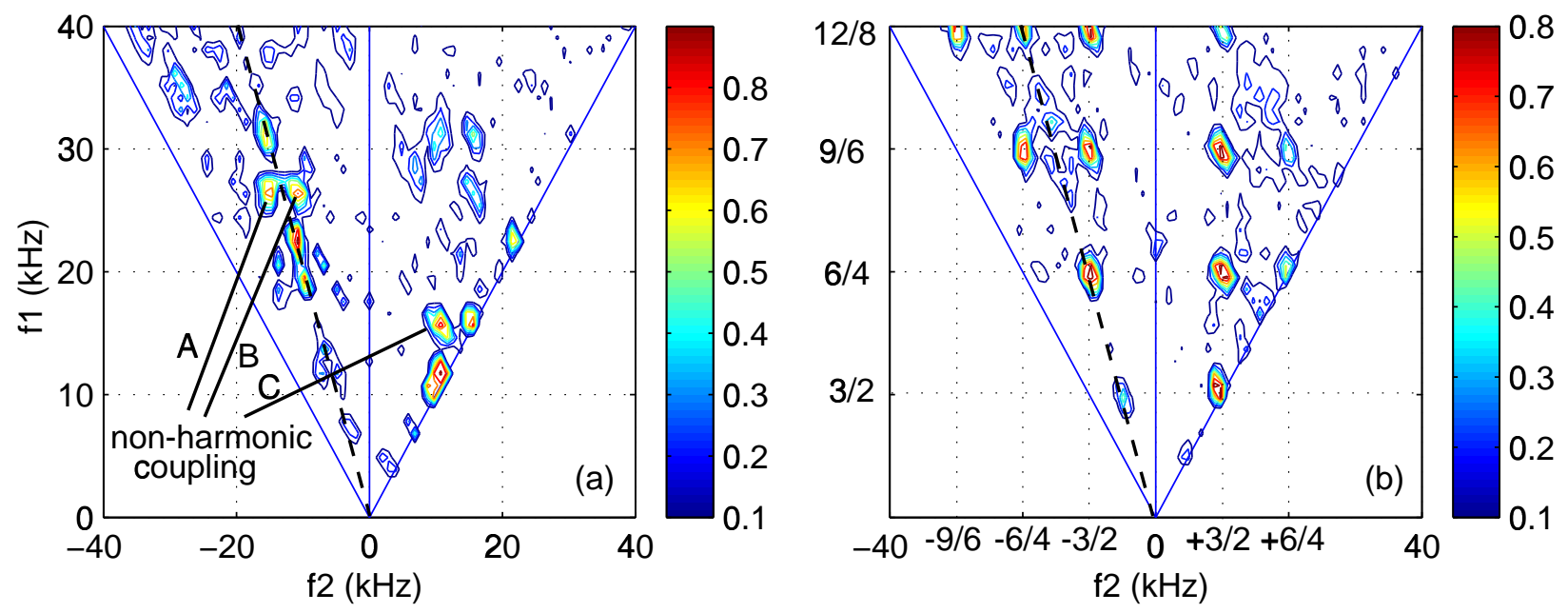

Figure 4. Bispectral analysis of JET case shown in Fig. 3, with the same scale for both plots: (a) between 18.75s and $18.82 \mathrm{~s}$, immediately after the sawtooth crash. The peaks related to the non-harmonic nonlinear coupling between the $3 / 2(12 \mathrm{kHz}), 4 / 3(15 \mathrm{kHz})$ and the $7 / 5(27 \mathrm{kHz})$ are marked by the arrows (A relates to $\frac{7}{5}-\frac{4}{3}$, B to $\frac{7}{5}-\frac{3}{2}$ and $\mathrm{C}$ to $\left.\frac{4}{3}+\frac{3}{2}\right)$. The peaks on the black dashed lines are related to harmonic coupling. (b) between $18.99 \mathrm{~s}$ and $19.06 \mathrm{~s}$, only multiple harmonic couplings are observed as obtained from the mode labels on the yand $\mathrm{x}$-axis. The time intervals are marked in Fig. 3.

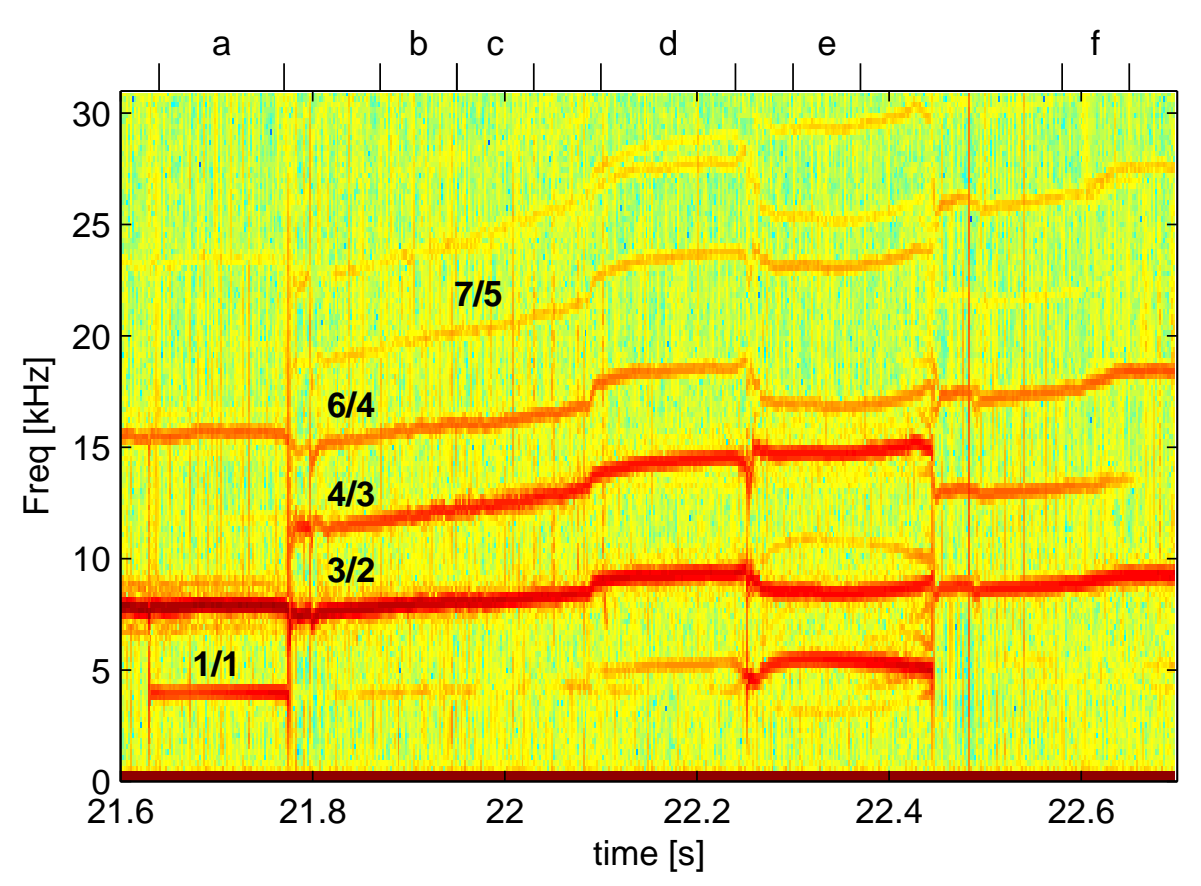

Figure 5. Spectrogram between 21.6s and 22.7s of the JET case, \#53290, corresponding to Fig. 10 of Ref. [10]. The interval used for the bispectrum analysis shown in Fig. 6 are also marked. 

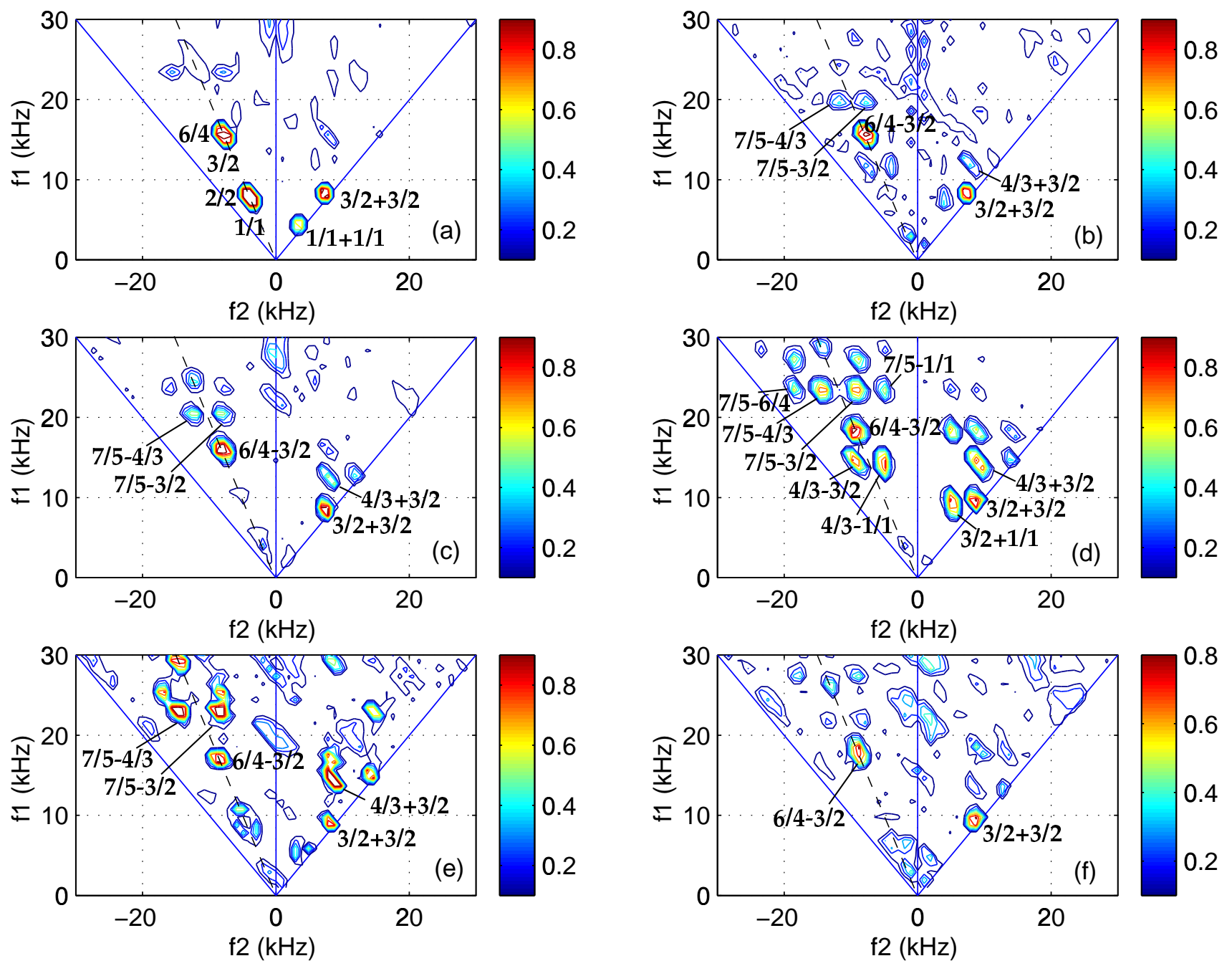

Figure 6. Bispectral analysis of the case shown in Fig. 5: (a) just before the sawtooth crash, between $21.64 \mathrm{~s}$ and $21.77 \mathrm{~s}$ (b) after first sawtooth crash, between $21.87 \mathrm{~s}$ and $21.95 \mathrm{~s}$ (c) between $21.95 \mathrm{~s}$ and $22.03 \mathrm{~s}$ (d) between $22.1 \mathrm{~s}$ and $22.24 \mathrm{~s}$ (e) between $22.30 \mathrm{~s}$ and $22.37 \mathrm{~s}$ (f) After the second sawtooth crash, between $22.58 \mathrm{~s}$ and $22.65 \mathrm{~s}$. The respective time intervals are marked in Fig. 5 

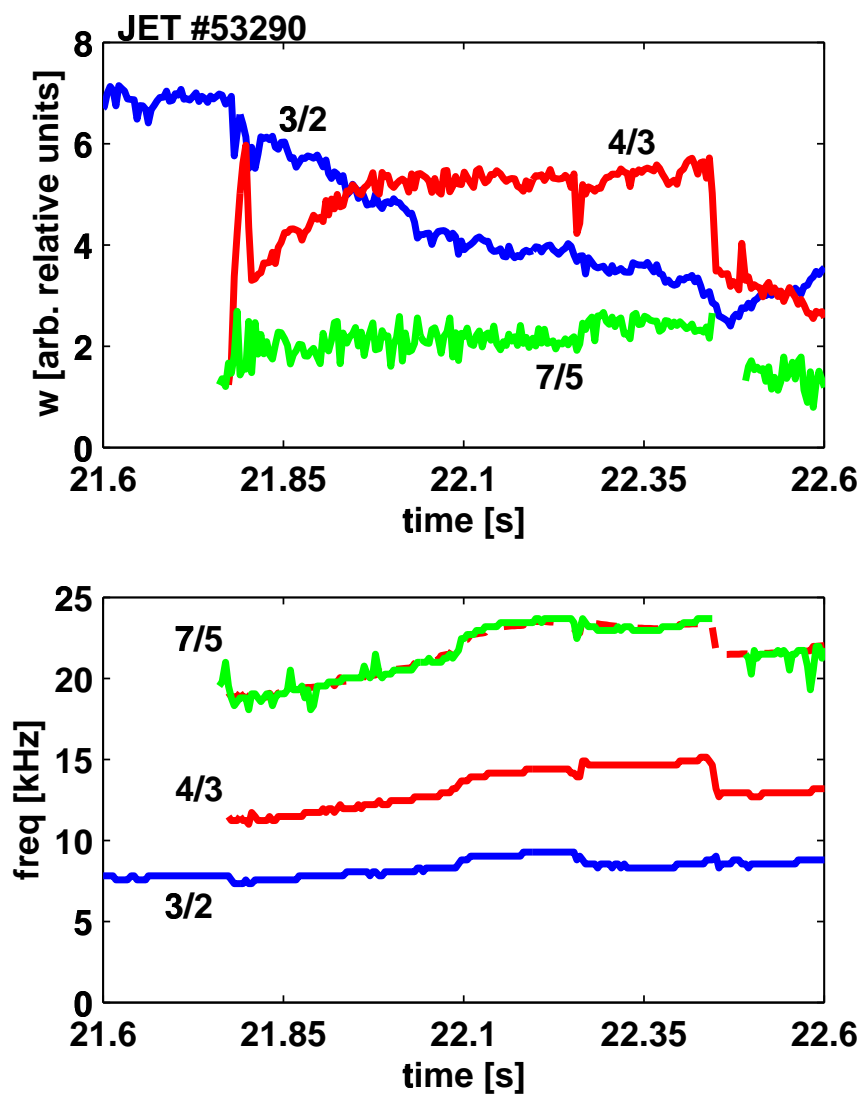

Figure 7. (a) Time evolution of $w \sim \sqrt{\delta B}$ for the three modes interacting in JET \#53290, Fig. 6. Each curve is scaled by an arbitrary constant as the $4 / 3$ and $7 / 5$ island width have not been calibrated.

(b) Time evolution of the modes frequencies. The dashed line marks the sum of the $3 / 2$ and $4 / 3$ frequencies and is exactly aligned with the $7 / 5$ frequency whenever the latter has a measurable island width. 